Author(s): Francisco Antunes

Article title: Semantic web and decision support systems

Article no: TJDS 1087293

Enclosures: 1) Query sheet

2) Article proofs

Dear Author,

1. Please check these proofs carefully. It is the responsibility of the corresponding author to check these and approve or amend them. A second proof is not normally provided. Taylor \& Francis cannot be held responsible for uncorrected errors, even if introduced during the production process. Once your corrections have been added to the article, it will be considered ready for publication.

Please limit changes at this stage to the correction of errors. You should not make trivial changes, improve prose style, add new material, or delete existing material at this stage. You may be charged if your corrections are excessive (we would not expect corrections to exceed 30 changes).

For detailed guidance on how to check your proofs, please paste this address into a new browser window: http://journalauthors.tandf.co.uk/production/checkingproofs.asp

Your PDF proof file has been enabled so that you can comment on the proof directly using Adobe Acrobat. If you wish to do this, please save the file to your hard disk first. For further information on marking corrections using Acrobat, please paste this address into a new browser window: http:// journalauthors.tandf.co.uk/production/acrobat.asp

2. Please review the table of contributors below and confirm that the first and last names are structured correctly and that the authors are listed in the correct order of contribution. This check is to ensure that your name will appear correctly online and when the article is indexed.

\begin{tabular}{|c|c|c|c|c|}
\hline Sequence & Prefix & Given name(s) & Surname & Suffix \\
\hline 1 & & Francisco & Antunes & \\
2 & & Manuela & Freire & \\
3 & & João Paulo & Costa & \\
& & & & \\
\hline
\end{tabular}


Queries are marked in the margins of the proofs, and you can also click the hyperlinks below. Content changes made during copy-editing are shown as tracked changes. Inserted text is in red font and revisions have a red indicator $\curlywedge$. Changes can also be viewed using the list comments function. To correct the proofs, you should insert or delete text following the instructions below, but do not add comments to the existing tracked changes.

\section{AUTHOR QUERIES}

\section{General points:}

1. Permissions: You have warranted that you have secured the necessary written permission from the appropriate copyright owner for the reproduction of any text, illustration, or other material in your article. Please see http://journalauthors.tandf.co.uk/permissions/usingThirdPartyMaterial.asp.

2. Third-party content: If there is third-party content in your article, please check that the rightsholder details for re-use are shown correctly.

3. Affiliation: The corresponding author is responsible for ensuring that address and email details are correct for all the co-authors. Affiliations given in the article should be the affiliation at the time the research was conducted. Please see http://journalauthors.tandf.co.uk/preparation/writing.asp.

4. Funding: Was your research for this article funded by a funding agency? If so, please insert 'This work was supported by <insert the name of the funding agency in full>', followed by the grant number in square brackets '[grant number $\mathrm{xxxx}$ ]'.

5. Supplemental data and underlying research materials: Do you wish to include the location of the underlying research materials (e.g. data, samples or models) for your article? If so, please insert this sentence before the reference section: 'The underlying research materials for this article can be accessed at $<$ full link $>/$ description of location [author to complete]'. If your article includes supplemental data, the link will also be provided in this paragraph. See $<\mathrm{http}$ :/

journalauthors.tandf.co.uk/preparation/multimedia.asp $>$ for further explanation of supplemental data and underlying research materials.

6. The CrossRef database (www.crossref.org/) has been used to validate the references. Changes resulting from mismatches are tracked in red font.

\begin{tabular}{|c|c|}
\hline AQ1 & Please provide the missing department for Freire affiliation. \\
\hline AQ2 & $\begin{array}{l}\text { The sentence "...of an elementary sentence..." has been changed to "...of a simple } \\
\text { sentence...". Please check the change conveys the intended meaning or amend. }\end{array}$ \\
\hline AQ3 & $\begin{array}{l}\text { The reference 'Kousetti et al. (2008)' is cited in the text but is not listed in the } \\
\text { references list. Please either delete in-text citation or provide full reference details } \\
\text { following journal style } \\
\text { [http://www.tandf.co.uk/journals/authors/style/reference/tf_APA.pdf]. }\end{array}$ \\
\hline AQ4 & $\begin{array}{l}\text { The sentence "Rather than a completely different approach ..." has been changed } \\
\text { to "Rather than representing a completely different approach ...". Please check the } \\
\text { change conveys the intended meaning or amend. }\end{array}$ \\
\hline AQ5 & $\begin{array}{l}\text { The sentence "...than one made in presence" has been changed to "...than dis- } \\
\text { course that occurs in person". Please check the change conveys the intended mean- } \\
\text { ing or amend. }\end{array}$ \\
\hline AQ6 & The disclosure statement has been inserted. Please correct if this is inaccurate. \\
\hline AQ7 & Please provide missing details for the 'Kousetti et al. (2008)' references list entry. \\
\hline
\end{tabular}




\begin{tabular}{|c|c|}
\hline AQ8 & Please provide missing city for the 'Muysken (2000)' references list entry. \\
\hline AQ9 & Please provide missing editor name for the 'Necula (2011)' references list entry. \\
\hline AQ10 & $\begin{array}{l}\text { Please provide missing city for the 'Nonaka \& Takeuchi (2000)' references list } \\
\text { entry. }\end{array}$ \\
\hline AQ11 & Please provide missing details for the 'Padula et al. (2009)' references list entry. \\
\hline AQ12 & Please provide missing details for the 'Pattal et al. (2009)' references list entry. \\
\hline AQ13 & $\begin{array}{l}\text { Please provide missing page numbers for the 'Robu et al. (2009)' references list } \\
\text { entry. }\end{array}$ \\
\hline AQ14 & Please provide missing details for the 'Rodriguez et al. (2007)' references list entry. \\
\hline AQ15 & $\begin{array}{l}\text { Please provide missing editor name for the 'Surowiecky (2004)' references list } \\
\text { entry. }\end{array}$ \\
\hline AQ16 & $\begin{array}{l}\text { Figure 1: Please spell out all abbreviations used in the figure in full at first mention. } \\
\text { Some of the text in the figure is obscured by a bullseye - if this is not intentional, } \\
\text { please edit the figure to correct this. Please confirm permission has been obtained } \\
\text { to publish the image in Figure } 1 \text {. Please provide details of the permission informa- } \\
\text { tion, to be included in the figure caption. The reference "http://www.w3.org" is } \\
\text { cited in the text but is not listed in the reference list. Please either delete the in-text } \\
\text { citation or provide full reference details following journal style, and then cite it here } \\
\text { using the author/organization name and year. }\end{array}$ \\
\hline AQ17 & Please spell out all abbreviations in Table 1 in full. \\
\hline AQ18 & Please spell out all abbreviations in Table 2 in full. \\
\hline
\end{tabular}




\section{How to make corrections to your proofs using Adobe Acrobat/Reader}

Taylor \& Francis offers you a choice of options to help you make corrections to your proofs. Your PDF proof file has been enabled so that you can mark up the proof directly using Adobe Acrobat/Reader. This is the simplest and best way for you to ensure that your corrections will be incorporated. If you wish to do this, please follow these instructions:

1. Save the file to your hard disk.

2. Check which version of Adobe Acrobat/Reader you have on your computer. You can do this by clicking on the "Help" tab, and then "About".

If Adobe Reader is not installed, you can get the latest version free from http:/get.adobe.com/ reader/.

3. If you have Adobe Acrobat/Reader 10 or a later version, click on the "Comment" link at the righthand side to view the Comments pane.

4. You can then select any text and mark it up for deletion or replacement, or insert new text as needed. Please note that these will clearly be displayed in the Comments pane and secondary annotation is not needed to draw attention to your corrections. If you need to include new sections of text, it is also possible to add a comment to the proofs. To do this, use the Sticky Note tool in the task bar. Please also see our FAQs here: http://journalauthors.tandf.co.uk/production/index.asp.

5. Make sure that you save the file when you close the document before uploading it to CATS using the "Upload File" button on the online correction form. If you have more than one file, please zip them together and then upload the zip file.

If you prefer, you can make your corrections using the CATS online correction form.

\section{Troubleshooting}

Acrobat help: http://helpx.adobe.com/acrobat.html

Reader help: http://helpx.adobe.com/reader.html

Please note that full user guides for earlier versions of these programs are available from the Adobe Help pages by clicking on the link "Previous versions" under the "Help and tutorials" heading from the relevant link above. Commenting functionality is available from Adobe Reader 8.0 onwards and from Adobe Acrobat 7.0 onwards.

Firefox users: Firefox's inbuilt PDF Viewer is set to the default; please see the following for instructions on how to use this and download the PDF to your hard drive: http://support.mozilla.org/en-US/kb/ view-pdf-files-firefox-without-downloading-them\#w_using-a-pdf-reader-plugin 


\title{
Semantic web and decision support systems
}

\author{
${ }^{a}$ Management and Economics Department, Beira Interior University and INESCC, Coimbra, \\ Portugal; ${ }^{b}$ INESC Coimbra, Coimbra, Portugal; ${ }^{c}$ Faculty of Economics, Coimbra University and \\ INESCC, Coimbra, Portugal
}

(Received 4 July 2013; accepted 27 August 2013)

\begin{abstract}
Semantic Web technologies are intertwined with decision-making processes. In this paper the general objectives of the semantic web tools are reviewed and characterised, as well as the categories of decision support tools, in order to establish an intersection of utility and use. We also elaborate on actual and foreseen possibilities for a deeper integration, considering the actual implementation, opportunities and constraints within the context of decision-making. We conclude that a broader or generalised Semantic Web integration in the decision support community is still a work in progress and much remains to be done.
\end{abstract}

Keywords: semantic web; decision-making; group decision support; web evolution

\section{Introduction}

Web 1.0 is known as an early stage of the conceptual evolution of the World Wide Web, where users simply acted as mere publishers and consumers of content, as web page information was closed to external editing. Rather than a specific technology update or specification, the Web 2.0 core was a transformation in the way web pages were made and used. The term 'Web 2.0' is used to describe applications that take advantage of the network nature of the Web, encourage the participation of community members, are inherently social and opened, aiming at enhancing information sharing as well as fostering collaboration (Abramowicz, Fensel, \& Frank, 2010). The popularity of the term Web 2.0, that echoed the common people, called for a set of technology that puts users at the centre of the applications (O'Reilly, 2005, 2006). There is a clear change in how technology is used: the application is what users make of it, and so, the more users you have, the better the application becomes. It involves a major conceptual shift in how information is created, validated, managed, shared and consumed. Essentially, Web 2.0 applications add a multitude of users who are responsible for all of these information management activities. The term Web 2.0 classifies applications such as Wikipedia, Facebook, YouTube, weblogs, microblogging, social bookmarking services, etc., which are also termed 'social software' (Lai \& Turban, 2008; Richter, Riemer, \& Brocke, 2011). Although social software and decision-making are clearly related in terms of their objectives (enhanced collaboration, information sharing and knowledge acquisition, according to Power \& Phillips-Wren, 2011), they present major differences from ${ }_{\curlywedge}$ an organisational ${ }_{\curlywedge}$ perspective. Traditional group decision-making

\footnotetext{
*Corresponding author. Email: francisco.antunes@ubi.pt
} 
presents a 'top-down' approach, usually designed to deliberately guide the interactions of groups in decision-making processes, while in social software, users, on the public internet, generate the content and define both the rules and the reasons for usage (Boyd, 2006) - and thus the social software approach is essentially 'bottom up'. This approach brings new possibilities in involving a massive collectiveness (of, for example, potential and actual consumers, voters, subscribers, fans, etc.) into decision processes (like product development, policy definition, content selection, hiring a coach, etc.), especially during its early stages - intelligence and design phases, as defined by Simon (1977). As traditional group decision-making tools do not encompass this situation, it creates a gap between traditional decision support systems, which usually cover the sequential support of all decision-making tasks, and using Web 2.0 tools for decision-making. As Web 2.0 is based on different tools (Nagle \& Pope, 2013), depending on the problem in hand, organising and integrating the generated information might constitute a major problem. In this paper, we argue that such a problem can be mitigated by intersecting Web 2.0 and semantic web technologies.

The term 'semantic web' (Berners-Lee, Hendler, \& Lassila, 2001) is considered by many an evolution of Web 2.0 - hence the term 'Web 3.0' (Lassila \& Hendler, 2007), though there are many detractors of this expression - means a set of technologies that includes ontologies, software agents and rules of logic. These technologies can greatly improve the ability to connect and automatically organise the content of information spread across multiple pages or sites (Kousetti, Millard, \& Howard, 2008), created within the intelligence phase, and to structure and represent such information, thus aiding the design and choice phases of decision processes (Simon, 1977).

We will make a brief initial review of the general objectives and technologies proposed with the implementation of the semantic web, to later evidence how to combine its actual implementation to enhance opportunities and tackle perceived constraints within the context of decision-making.

\section{Semantic Web technology}

While Web 2.0 focuses on humans, mostly by providing efficient platforms for information sharing, the semantic web focuses on machines, by providing machineprocessable information (Abramowicz et al., 2010), especially through semantic languages and tools for ontologies and metadata management (Padula, Reggiori, \& Capetti, 2009).

This section provides a brief review of the main technical aspects of the semantic web, for later review and integration with the decision-making process and its tools.

\subsection{A vision for the future}

The semantic web (according to Berners-Lee et al., 2001) will enable machines to comprehend semantic documents and data, not human speech and writings. Moreover, the semantic web, in naming every concept simply by a uniform resource identifier (URI), should express, seamlessly, new concepts that people invent. This unifying logical tanguage should enable these concepts to be progressively linked into a universal web, thus opening up the knowledge and workings of humankind to meaningfull analysis by software agents. Therefore, the challenge is to provide a language that expresses both data and rules for reasoning about the data and that allows rules from any existing knowledge-representation system to be exported onto the Web. 


\subsubsection{XML}

The base of Web 3.0 for exposing data to applications is the Extensible Markup Language (XML), which lets everyone create their own tags - hidden labels that annotate web pages or sections of text on a page. Scripts, or programs, can make use of these tags in sophisticated ways, but the script writer has to know the reason for which the page writer uses each tag. In short, XML allows users to add arbitrary structure to their documents, but says nothing about what the structures mean (Berners-Lee et al., 2001).

\subsubsection{RDF}

Meaning is expressed by Resource Description Framework (RDF), which encodes it in sets of triples that use URIs to name the relationship between things as well as the two ends of the link, each triple being rather like the subject, verb and object of a simple sentence (Berners-Lee et al., 2001). This simple model allows structured and semi-structured data to be mixed, exposed and shared across different applications. The resulting linking structure forms a directed, labelled graph, where the edges represent the named link between two resources, represented by the graph nodes. This graph view

20 is the easiest possible mental model for RDF, and is often used in easy-to-understand visual explanations.

\subsubsection{Queries}

With SPARQL (a recursive acronym for SPARQL Protocol and RDF Query Language), a query language for RDF data, applications can access native graph-based RDF stores and extract data from traditional databases (Hendler, 2009). Technically, SPARQL queries are based on triple patterns. These triple patterns are similar to RDF triples, except that one or more of the constituent resource references are variables. A SPARQL engine would return the resources for all triples that match these patterns.

The semantic web is a 'web of data' - of dates and titles and part numbers and any other data one might conceive of. To make it a reality, a huge amount of Web-available data, in a standard format, reachable and manageable by semantic web tools, is needed. SPARQL is intended to integrate disparate databases (domain-limited or specific databases - relational, XML, HTML, etc.) so that one query spans (seamlessly and on the fly) through several data sets to deliver targeted results (Lassila \& Hendler, 2007). This collection of interrelated data sets on the Web can also be referred to as linked data.

\subsubsection{Ontologies}

On the semantic web, vocabularies or ontologies define the concepts and relationships (also referred to as 'terms') used to describe and classify terms that can be used in a particular application, characterise possible relationships and define possible constraints on using those terms. The most typical kind of ontology for the Web has a taxonomy and a set of inference rules. The taxonomy defines classes of objects and relations among them (Berners-Lee et al., 2001). Web Ontology Language (OWL) is a language that can play a main role in the applications of Web 3.0. OWL and RDF are much the same thing, but OWL is a stronger language with greater machine interpretability than RDF. OWL is built on the top of RDF but comes with a larger vocabulary and stronger 
syntax than RDF does (Pattal, Yuan, \& Jianqiu, 2009), being the basis for implementing inference techniques on the semantic web.

Inference rules in ontologies can be characterised by discovering new relationships among terms. This means that automatic procedures can generate new relationships based on the data and based on some additional information in the form of ontologies. Although the computer doesn't truly 'understand' any of these relationships, it can manipulate the terms much more effectively in ways that are useful and meaningful to the human user (Berners-Lee et al., 2001). Inference is also intended to improve data integration and handling possible data inconsistencies on the Web, by seamlessly analysing data content.

\subsection{The actual Semantic Web}

In spite of the earlier vision for a future with Web 3.0 (Berners-Lee et al., 2001), the problem is that a complete re-annotation of the Web is a massive undertaking. As an alternative, many researchers take a very different approach to the semantic web. Rather than calling for an overhaul of Web formats, and the massive effort of using semantic web tools (not to be expected), which would involve hundreds of thousands of independent sites, they are building software agents that can better understand web pages, as they exist today. Instead of waiting for additional information and for more 'machine-understandable' web pages, the alternative is developing improved software agents for information retrieval and natural language processing.

\subsubsection{NLP and IE}

Naturallanguage processing/programming (NLP) is a field of computer science, artificial intelligence (AI) and linguistics that regards the interactions between computers and human (natural) languages. NLP is a theoretically motivated range of computational techniques for analysing and representing naturally occurring texts at one or more levels of linguistic analysis for the purpose of achieving human-like language processing for a range of tasks or applications (Acharya \& Parija, 2010). NLP is also an ontology-assisted way of programming in terms of natural language sentences.

NLP and information extraction (IE) seek to deduce rules or a domain model out of texts. Such efforts include a strong machine-learning component, in addition to the NLP component. The knowledge base they hope to extract is frequently designed to drive an expert system, or case-based reasoner or knowledge-driven decision support systems (Cowie \& Lehnert, 1996).

Information extraction (IE) is a more limited task than 'full text understanding', as full text understanding represents all of the information in a text. In contrast, IE delimits, in advance, the semantic range of the output. It identifies specific pieces of information (data) in an unstructured or semi-structured textual document (e.g. a web page) and transforms unstructured information in a corpus of documents or web pages into a structured database (Acharya \& Parija, 2010).

\subsubsection{Agents}

In AI, an intelligent agent (IA) is an autonomous entity, which observes through sensors, acts upon an $\hat{\wedge}$ environment using actuators and directs its activity towards achieving rational goals (Russell \& Norvig, 2010). Intelligent agents may also learn or 
use knowledge to achieve their goals, ranging from very simple or very complex (a thermostat is an intelligent agent if it has a rational goal, as is a human being, as is a community of human beings working together towards a goal, as described in Franklin \& Graesser, 1996). IAs in AI are closely related to agents in economics, and versions of the intelligent agent paradigm are studied in cognitive science, ethics and the philosophy of practical reason, as well as in many interdisciplinary socio-cognitive modelling $_{\curlywedge}$ and computer social simulations. Semantics enable agents to locate others (or automated Web-based services) that will perform a required function, and to describe precisely what function they carry out and what input data are needed. The real power of the semantic web will be realised when people create many programs that collect Web content from diverse sources, process the information and exchange the results with other programs (Berners-Lee et al., 2001). Nevertheless, the effectiveness of such software agents can only achieve its full potential when more 'machine-readable' Web content and automated services (including other agents) become available.

\section{Decision-making and Semantic Web}

The semantic web shares many goals with decision support systems (DSS), namely by being able to precisely interpret information, in order to deliver relevant, reliable and accurate information to a user, but also by presenting an enhanced ability to connect and automatically organise the content of information spread across multiple pages or sites (Kousetti et al., 2008). This ability has implications for decision-making support, namely fulfilled and unfulfilled promises derived from the earlier vision of the semantic web and research opportunities. We will address them in the following sub-sections.

\subsection{Intersecting DSS and Web technologies}

We can accept the categories of decision support tools (as established in Arnott \& Pervan, 2005, 2008), based on their main objectives:

(1) Personal decision support systems (PDSS): usually small-scale systems that are developed for one manager, or a small number of independent managers, to support a decision task;

(2) Group support systems (GSS): the use of a combination of communication and decision support technologies to facilitate the effective working of groups;

(3) Negotiation support systems (NSS): decision support tools where the primary focus of the group work is the negotiation between opposing parties;

(4) Intelligent decision support systems (IDSS): the application of AI techniques to decision support;

(5) Knowledge management-based decision support systems (KMDSS): systems that support decision-making in aiding knowledge storage, retrieval, transfer and application, by means of supporting individual and organisational memory and inter-group knowledge access;

(6) Data warehousing (DW): systems that provide the large-scale data infrastructure for decision support;

(7) Enterprise reporting and analysis systems (ER): enterprise-focused decision support technologies, including executive information systems (EIS), business intelligence $(\mathrm{BI})$ and, more recently, corporate performance management systems (CPM). 
The semantic web data can be used in several ways to process and share information (Blomqvist, 2013), namely, in a DSS context:

(1) Information integration (several data sources, different formats, external data sources, high change rate, exchangeable data sources);

(2) Information filtering and selection (several large data sources, different tasks and roles of users, abstraction);

(3) Information extension, exploration, and explanation (data may be missing in internal sources, user explanations, browsing relations between data, drill-down of information);

(4) Information interpretation, event detection and prediction (large data sources, high change rate of data, abstraction and aggregation, situation detection, 'realtime' data, data analysis);

(5) Information tracking and post-event analysis (large data sources, abstraction and aggregation, situation detection, post-session evaluation and session followup, provenance);

(6) Models and model evolution (different changing data formats, external data sources, changing user tasks and views, model-based analysis, relations amongst information, browsing and linking);

(7) Sharing decisions (trust, provenance, accountability, user created data, interaction between users, delegation).

The feature categories span across the different DSS and semantic web tools (presented in section 2), even though they are not always present or do not always bear the same importance. We can match the utility of each semantic web tool to information processing and sharing against each decision support tool category; the intersection of tools and feature categories is depicted in Table 1. For instance, RDF can be very useful in GSS for (1) information integration, (3) information extension, exploration and explanation and (7) sharing decisions.

DSS can be viewed from several different perspectives (Arnott \& Pervan, 2005; Power, Sharda, \& Kulkarni, 2007; Turban, Aronson, \& Liang, 2005), namely:

AQ17 Table 1. Intersection of the semantic web and decision support.

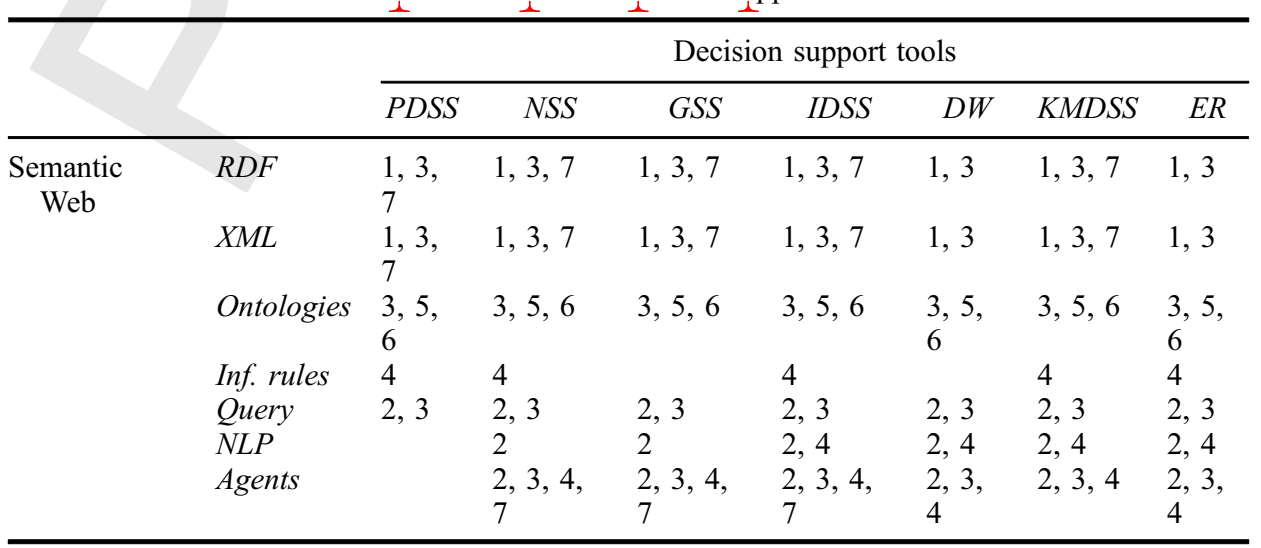


(1) Model-driven

(2) Communication-driven

(3) Knowledge-driven

(4) Data-driven.

We can trace those perspectives to Web evolution, according to their intrinsic purposes, as represented in Table 2. It is easy to realise that PDSS are much more related to producing content than disseminating such content, while NSS and GSS naturally involve a multitude of users (even though bearing different objectives). Knowledge-driven and data-driven DSS can benefit the most from semantic web features, as it provides enhanced content relationships with the possibility for greater retrieval accuracy.

The evolution of web technologies creates new possibilities for group decision support, bringing the possibility to access the potential of the 'wisdom of the crowds' (the term is defined by Surowiecky, 2004), although using different tools, a situation that might hinder their integration. The next subsection expresses how semantic web tech20 nologies can overcome this problem.

\subsection{Group Decision Support}

Regarding the creation of information, and contrarily to the traditional group decisionmaking 'top-down' approach, usually involving small groups, Web 2.0 stands for a 'bottom-up' approach where information is produced by mass collaboration of people

25 (though it does not mean that mass collaboration will substitute for the decision-making of small groups) that create, update and share knowledge on a regular basis (Gehrke \& Wolf, 2010), which constitutes a very distinct approach not only from PDSS, but also from traditional GSS, in terms of argumentation process, sequential support of activities and people involved.

30 Though this 'bottom-up' approach can be very useful for generating information, it is usually done by using distinct tools, like blogs, wikis, discussion groups, etc. (for a more detailed view on the subject see, for instance, Turban, Liang, \& Wu, 2011), rather than using a single GDSS that usually supports all group decision-making tasks, thus making information more difficult to integrate. This gap can be filled using semantic 35 web techniques like ontologies, software agents and social classification of information

AQ18 Table 2. Web stages and their adequacy regarding decision-making tools.

\begin{tabular}{lllll}
\hline & & \multicolumn{3}{c}{ Web } \\
\cline { 3 - 5 } Power et al., 2007 & Arnott \& Pervan, 2005, 2008 & 1.0 & 2.0 & 3.0 \\
\hline Model-driven & PDSS & +++ & + & + \\
& NSS & ++ & +++ & ++ \\
Communications-driven & GSS & ++ & +++ & ++ \\
Knowledge-driven & IDSS & ++ & + & +++ \\
& KMDSS & ++ & ++ & +++ \\
Data-driven & DW & ++ & + & +++ \\
& ER & ++ & + & +++ \\
\hline${ }^{+++}$Excellent fit. & & & & \\
${ }^{++}$Adequate fit. & & & &
\end{tabular}


relevance (through registered classifications performed by past information users, according to their perceived relevance). These techniques provide a larger spectrum of possibilities in searching and recovering relevant information (Antunes \& Costa, 2012). Compared to ontologies, 'folksonomies' offer greater flexibility and adaptability in organising information, and users do not need to agree on a detailed tag hierarchy and taxonomy, though it implies that each user can create a separate set of tags that would then need to be disambiguated, using specific ontologies to be created or a combination with existing ontologies. Folksonomies may also suffer from ambiguity regarding the meaning of the tags and lack of semantics, for example, synonyms. Moreover, a coherent categorisation scheme when using folksonomies can become difficult to achieve, because their contributors do not operate under a centralised controlling vocabulary, though empirical work shows the emergence of stable collective consensus around the categorisation of information driven by 'tagging' behaviours (Robu, Halpin, \& Shepherd, 2009).

Based on the above-mentioned, we have, on one hand, tagging tools that require specific computer skills with low ease of use and, on the other hand, users who do not willingly spend time on this extra work just because it might bring them future unspecified benefits or, more altruistically, improve the development of the semantic web, thus contributing to a feeling of uselessness of the process. Therefore, and according to the Technology Acceptance Model and its extensions (Venkatesh \& Davis, 2000), in spite of the fact that people seamlessly create and disseminate information through social media, the intention to individually add any further annotations to content seems compromised (at least until they have better tools to do so). According to this situation, the use of software agents and NLP seems appropriate to perform an automatic processing of the dynamic and massive amount of information encompassed in social media, at least until technology takes full advantage of folksonomies.

Another issue largely different from traditional GDSS is decision-making trust (i.e. that the people involved in the decision are fit or adequate to the process). When in the presence of social-networked decision-making, the notion of decision-making trust must be made explicit (Rodriguez et al., 2007), as friendship, per se (seldom the reason for linking users), does not identify people as good decision-makers. The familiarity with the technological features and communication tools of social media, or satisfaction with past interactions with other community members, are much more important antecedents for online trust than mere acquaintance or friendship (Grabner-Kräuter, 2009). Nevertheless, semantic web reasoners, agents, and other automated systems can enhance trust judgments, by enabling the detection of related statements, and whether they are contradictory (Gil \& Artz, 2007), thus enhancing the trustworthiness of web content by analysing their semantic relationship (Gao, 2010) and also to structure web content in order to extract or perceive the implicit argumentation liaisons.

\subsection{Decision process}

AQ4 Rather than representing a completely different approach, the concepts of semantic web complement Web 1.0 and Web 2.0 decision-making quite well. Complementary to Web 1.0 , the use of formats such as RDF and OWL and the incorporation of linked data in DSS applications are intended to access, extract and integrate data from $\hat{e}$ existing data sources, which is like data warehousing approaches to database integration but with new formats. 
The unstructured nature of decision-making is very well suited for the ad hoc nature of social networking based on different tools (Nagle \& Pope, 2013), depending on the problem at hand, with users organising information according to the problem itself, rather than a preformatted way of collaboration that might not be appropriate for every single case. This situation fits rather well the early stages of the decision-making process (as defined by Simon, 1977), and semantic web technologies are, therefore, intertwined with it. In the following sub-sections, we will elaborate on actual and foreseen possibilities for a deeper integration.

\subsubsection{Intelligence}

During the intelligence phase, problem finding, analysis and definition occur and divergence is supported through the generation of alternatives. As a group evaluates the alternatives, the convergence process evolves.

Input data in the decision-making model is changing (from personal to mass collab15 oration and integration of external data), although the semantic web is not a decision-

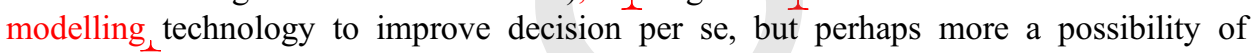
integrating data (Necula, 2011). Therefore, improving the search, enhancing knowledge sharing and integrating the available, though heterogeneous, databases and semantic interoperability, will reveal implicit information that usually would remain undiscov20 ered, thereby resulting in suboptimal decisions (Necula, 2012).

In the specific case of social media, the ontologies derived from folksonomies can give a machine-processable form to the social web's collective intelligence, enabling Web 2.0 search engines to deliver more advanced information retrieval options with better results. NLP and semantic web technologies could then be exploited to the advantage of DSS, namely by applying IE to populate semantic web data sets and to perform the automatic detection of arguments within group discourse (and from external data), for later analysis by a DSS. By interconnecting users' contributions, this process would enrich and produce a much more accurate information to be used in the intelligence phase. Nevertheless, the creation of folksonomies lacks tools that can 30 make this a seamless work (or at least very simplified or intuitive), making it a timeconsuming task.

\subsubsection{Design}

During the design phase, possible solutions to the problem are generated, usually followed by the merging of related ideas and the elimination of redundant or irrelevant ones, through a structuring process that might include the elicitation of criteria and their relative importance, as well as the indication of a value system.

One of the ways to support the design phase is based on a group of people debating (which can also be associated to the intelligence phase) and structuring their ideas in a virtual environment. The more structured versions of a group discourse allow a better understanding of the expressed points of view, as well as the logical sequence of the discussion itself, particularly when the deliberative support is provided to a large group or the discussion is active during a long period. Indeed, the incorporation of new people into an already-started decision meeting can be very difficult if previous discourse is not presented in a structured way.

45 The structured discourse can range from threaded plain text to a more structured presentation, usually sustained by argumentation theory. However, social media does 
not favour ${ }_{\curlywedge}$ this latter type of structure, or the generation of tags that can explicitly define used concepts, applied values or any types of quantitative or qualitative parameters. Accordingly, the use of $\mathrm{XML} / \mathrm{RDF}$ to structure the produced meeting content could alleviate this problem. Nevertheless, the generation of tagged content, which would be of enhanced utility in decision-making, requires computer skills that cannot be expected from all participants in all decision meetings. As in the intelligence phase, the lack of tools that can make this a seamless work prevents the automatic (or at least very simplified or intuitive) creation of the structured content, and makes it a time-consuming task.

Therefore (and according to Schneider, Groza, \& Passant, 2013), there is a need for ontologies that are suitable for representing informal social web arguments and ontologies that map between the social world and the argumentative world. Nevertheless, social media are understood as failing the criterion of 'argumentative discussions' as general Web 2.0 tools, since their argumentation support is considered to be peripheral (Schneider et al., 2013), as web discourse has different characteristics than discourse that occurs in person. In the first place, it requires new language forms to express emerging concepts (Bodomo, 2010). Second, it is a kind of discourse that privileges an informal language, as opposed to a more formal and structured language. A literature review shows a perceived difficulty in structuring social network data (Shum, Cannavacciuolo, De Liddo, Iandoli, \& Quinto, 2011). The writing style commonly used in these platforms has a pattern out of the ordinary that sometimes makes it incomprehensible to those who are not part of the conversation and/or that culture or context, thus making it very hard to make it 'machine-understandable'. This is because social actors often make mistakes in spelling and/or grammar, use abbreviations (e.g. ASAP $=$ as soon as possible), symbols (e.g. : $(=$ sad) 'stretch' words (e.g. 'nooooo'), or include links, images, audio and video (Bodomo, 2010; Georgalou, 2010). Moreover, normally dialogue does not contain many used words, since users tend to mix symbols (e.g. smileys). There are also dialogues that do not even contain text (only links and/or tags).

Another problem (described in Muysken, 2000) is the fact that a dialogue can be written in more than one language (code-mixing, which refers to all cases where lexical items and grammatical features from two languages appear in a sentence). As users can also omit much of speech, the discourse can become a written summary of what is meant, which might hinder the contextualisation of words and associated concepts. This means that these data are possibly tangled, incomplete and sometimes error-prone. In this case, semantics lie hidden in speech content created by the interveners. Even harder to grasp are the artifices of language such as rhetoric and wordplay (that turn out to be discursive strategies), and the origin, destination, intentions and reception of speech, which help to define how these interactions and respective arguments do come out. Herein lies the challenge to achieving its capture in order to be used by 'machines'. One way of doing this is by using formal models that capture arguments and convert implicit knowledge (concealed in discussions) to explicit knowledge (Nonaka \& Takeuchi, 1995).

In spite of the earlier considerations, and knowing that RDF triples consist of text encompassing relations between described entities, we can argue that semantic web tools will be able to transform the representation of a simple (unstructured) text into a representation that follows or is supported by one or more argumentation models. Such a process would follow: 
(1) The establishment/extraction of a taxonomy of elements contained in the text/ speech;

(2) The development of a specific ontology or the use of existing ontologies to relate the elements included in the taxonomy;

(3) The development of ontologies according to the intended argumentation models;

(4) A combination of step 2 and step 3.

The revelation of implicit attributes or argumentative properties can be achieved by IE/NLP techniques that could also build and associate different ontologies containing rules of the argumentative association derived from semantic terms (e.g. terms such as 'in support of', 'against', 'in favour of', etc.). Future social semantic web prototype tools for sense-making and argument-mapping could be tested for argumentation on some common debate topic in order to find a large audience of potential evaluators. It would also provide meaningful ways to discover new debate topics, and potentially record and share the outcome of these debates, making them potentially more savvy about argumentation schemes and similar abstractions (Schneider et al., 2013).

These processes combine the ease of use of social media for presenting, discussing and narrowing ideas (intelligence and design phases), while using AI tools (IE/NLP in particular) to structure the produced content (even though manual/human intervention is expected to some extent) and, thus, leading to the choice phase. This would be done by enabling a richer and more structured visualisation of the speech (for which visualisation analytics and tools are complementary to semantic web tools), namely by presenting the information according to different models of argumentation.

\subsubsection{Choice}

30 Choice involves divergent evaluation of the previous set of ideas and convergent selection, possibly following an iterative process.

Many DSS applications use ontologies and rules as a means for making the DSS 'intelligent' in some data analytics sense, in continuation of the expert systems tradi-

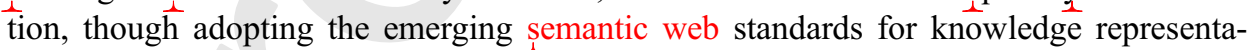
tion (Blomqvist, 2013). The use of an ontology of ontologies (according to Gaševic, Djuric, \& Devedžic, 2009) can facilitate collaboration by providing a unifying multiple-criteria decision analysis/aiding (MCDA) decision knowledge skeleton that can be used as a common and shared reference for a collaborative process. In addition, the deployment of service-oriented architectures (SOA), enhanced by semantic web tech40 nologies for sharing and accessing data, can apply semantic web technologies in peerto-peer networks, for facilitating offers in negotiation scenarios (Du, 2009).

\subsubsection{Implementation and evaluation}

Semantic web tools, namely ontologies, could also be applied to provide a follow-up on decisions after they are taken. This could become an excellent source for decision reconstruction and evaluation of the implemented choice (Antunes \& Costa, 2013). Unfortunately, the pervasiveness of ontologies in the Web is not yet a reality, as their creation involves a 'top-down process', which constantly requires disciplinary experts checking the evolution of the ontologies (Padula et al., 2009). 


\section{Final remarks}

Long gone is the idea that DSS were proprietary based and that a single package of software would suffice in supporting all the activities of personal and group decisionmaking. The pervasiveness of web technologies, especially regarding Web 2.0, made them globally available to be exploited in the major activities in the decision-making process (Turban et al., 2011), though not without a cost. In spite of the fact that the decision-making process still bears its original objectives (as proposed by Simon, 1977), the diversity of tools, formats and types of applications to support them enhance the decision agents' new opportunities for timely and better decisions more than ever before - at least theoretically.

What we conclude in this paper is that there is a gap between foretold promises in using social web tools to the benefit of decision agents and its actual possibilities, as integrating such tools and information poses many problems, especially as regards group support. To do so, we have argued that semantic web technologies need to be intertwined with the above-mentioned tools, especially for information integration and representation during the earlier phases of the decision process, and especially in the design phase, in which increasing amounts of produced information need to be processed properly and in a timely fashion. In Table 3, we present major opportunities and constraints for using semantic web over existing Web 2.0 solutions when used throughout the decision-making process.

We also conclude that the greatest obstacle to actual arrival of the semantic web into decision support mostly relies on the technologies that have to come together in order to make it seamless. Some argue that it is unrealistic to expect busy people and businesses to create enough metadata to make the semantic web work. The simple tagging used in Web 2.0 applications lets users spontaneously invent their own descriptions, which may or may not relate to anything else. However, the solution to this problem may simply rely on better tools for creating metadata, like the blogging and social-networking sites that have made building personal websites easy.

The first step towards a real semantic web-based decision-making environment is making data accessible through queries, with no AI involved. Although Web 2.0 tools provide fundamental technological support to tacit knowledge, Web 3.0 evolution will concern technological tools supporting explicit knowledge (as they are defined in

Table 3. Semantic web and the decision-making process.

\begin{tabular}{lllll}
\hline \multicolumn{5}{c}{ Decision process } \\
\hline Opportunities & Intelligence & \multicolumn{1}{c}{ Design } & \multicolumn{1}{c}{ Choice } & \multicolumn{1}{c}{$\begin{array}{l}\text { Implementation } \\
\text { and evaluation }\end{array}$} \\
\hline $\begin{array}{l}\text { integration and } \\
\text { interoperability }\end{array}$ & $\begin{array}{l}\text { Enhanced structuring } \\
\text { and argument } \\
\text { representation of } \\
\text { collaborative } \\
\text { discourse }\end{array}$ & $\begin{array}{l}\text { Collaborative } \\
\text { MCDAFacilitating } \\
\text { offers in } \\
\text { negotiation } \\
\text { scenarios } \\
\text { Requires } \\
\text { specialised } \\
\text { knowledge }\end{array}$ & $\begin{array}{l}\text { Follow-up on } \\
\text { decisions }\end{array}$ \\
& $\begin{array}{l}\text { Requires specialised } \\
\text { computer }\end{array}$ & $\begin{array}{l}\text { Ontologies are not } \\
\text { web- } \\
\text { pervasiveRequires } \\
\text { specialised } \\
\text { knowledge }\end{array}$ \\
\hline & $\begin{array}{l}\text { computer } \\
\text { skillsNeeds } \\
\text { seamless tools }\end{array}$ & $\begin{array}{l}\text { utilityNeeds } \\
\text { seamless tools }\end{array}$ & & \\
\hline
\end{tabular}

MCDA: multiple-criteria decision analysis/aiding. 


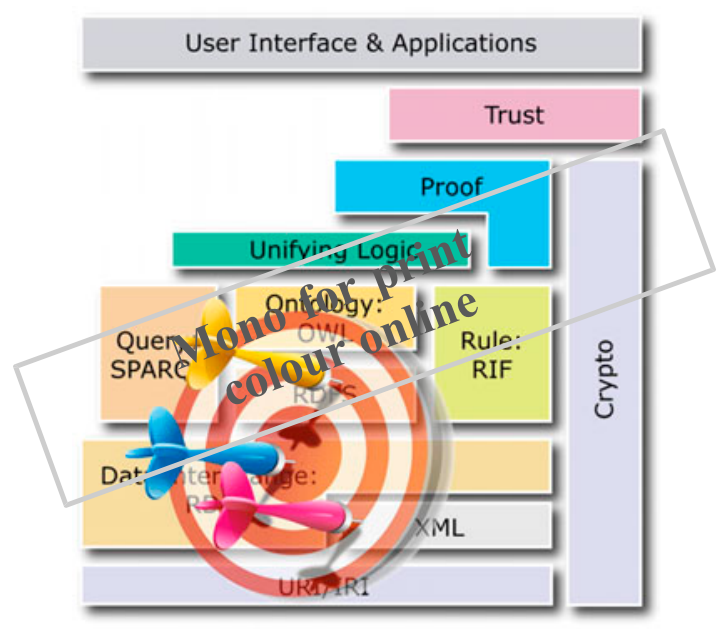

Figure 1. Targeted semantic web layer cake, regarding decision-making (source: adapted with AQ16 permission from http://www.w3.org).

Nonaka \& Takeuchi, 1995), where semantic interoperability, interoperable intelligent agents, ontology mapping and their progressive development appear to be overlapping areas requiring strong innovation (Padula et al., 2009).

The second step towards semantic web-based decision-making seems to be ontology mapping, as the number of publicly available ontologies increases steadily and as the semantic web grows (even though some argue its rhythm is not fast enough). The ontology-mapping process defines semantic bridges (and their interrelations) between a source and a target ontology, in order to exchange information between them.

If we combine the whole of the semantic web layer cake with the actual imple10 mentation in the decision-making context, we can see that it clearly marks a lesser target (Figure 1). It is easy to understand that a broader or generalised semantic web integration in the decision support community is still a work in progress, and much remains to be done.

\section{Acknowledgements}

15 This work was partially supported by the Portuguese Foundation for Science and Technology under project grant PEst-OE/EEI/UI308/2014.

\section{Disclosure statement}

AQ6 No potential conflict of interest was reported by the authors.

\section{References}

20 Abramowicz, W., Fensel, D., \& Frank, U. (2010). Semantics and web 2.0 technologies to support business process management. Business \& Information Systems Engineering, 1, 1-2. doi:10.1007/s12599-009-0089-5.

Acharya, S., \& Parija, S. (2010). The process of information extraction through natural language processing. International Journal of Logic and Computation (IJLP), 1, 40-51. 
Antunes, F., \& Costa, J. P. (2012). Integrating decision support and social networks. Advances in Human-Computer Interaction, 2012, 1-10. doi:10.1155/2012/574276.

Antunes, F., \& Costa, J. P. (2013). A decision reconstruction support model. International Journal of Information Technology \& Decision Making, 12, 309-333. doi:10.1155/2012/574276.

Arnott, D., \& Pervan, G. (2005). A critical analysis of decision support systems research. Journal of Information Technology, 20, 67-87.

Arnott, D., \& Pervan, G. (2008). Eight key issues for the decision support systems discipline. Decision Support Systems, 44, 657-672.

Berners-Lee, T., Hendler, J., \& Lassila, O. (2001). The semantic web. Scientific American, $34-43$.

Blomqvist, E. (2013). The use of semantic web technologies for decision support - a survey. Semantic Web, Pre-press, 1-25.

Bodomo, A. B. (2010). Computer-Mediated communication for linguistics and literacy: Technology and natural language education. Hershey, New York: Information Science Reference.

Boyd, D. M. (2006). The significance of social software. In T. N. Burg \& J. Schmidt (Eds.), BlogTalks Reloaded. Social Software - Research \& Cases. (pp. 15-30). Norderstedt, Germany: Books on Demand GmbH.

Cowie, J., \& Lehnert, W. (1996). Information extraction. Communications of the ACM, 39, $80-91$.

Du, T. C. (2009). Building an automatic e-tendering system on the Semantic Web. Decision Support Systems, 47, 13-21.

Franklin, S., \& Graesser, A. (1996). Is it an agent, or just a program?: A taxonomy for autonomous agents third international workshop on agent theories, architectures, and languages (pp. 21-35). Budapest, Hungary: Springer-Verlag.

Gao, Q. (2010). Towards trust in web content using semantic web technologies. In L. Aroyo, G. Antoniou, E. Hyvonen, A. T. Teije, H. Stuckenschmidt, L. Cabral \& T. Tudorache (Eds.), The Semantic Web: Research and Applications: Lecture Notes in Computer Science (Vol. 6089, pp. 457-461). Berlin: Springer-Verlag.

Gaševic, D., Djuric, D., \& Devedžic, V. (2009). Model driven engineering and ontology development (2nd ed.). Berlin: Springer.

Gehrke, N., \& Wolf, P. (2010). Towards audit 2.0 - a web 2.0 community platform for auditors $43 r d$ hawaii international conference on system sciences (CD-ROM). Kauai, Hawaii, USA: Computer Society Press.

Georgalou, M. (2010). "Pathfinding" discourses of self in social network sites. In R. Taiwo (Ed.), Handbook of Research on Discourse Behavior and Digital Communication: Language Structures and Social Interaction (pp. 39-62). Hershey, New York: IGI Global.

Gil, Y., \& Artz, D. (2007). Towards content trust of web resources. Web Semantics: Science, Services and Agents on the World Wide Web, 5, 227-239.

Grabner-Kräuter, S. (2009). Web 2.0 social networks: The role of trust. Journal of Business Ethics, 90, 505-522. doi:10.1007/s10551-010-0603-1.

Hendler, J. (2009). Web 3.0 emerging. Computer, 42, 111-113.

Kousetti, C., Millard, D. E., \& Howard, Y. (2008). Study of ontology convergence in a semantic wiki. In WikiSym08 2008 International Symposium on Wikis. Porto, Portugal.

Lai, L. S. L., \& Turban, E. (2008). Groups formation and operations in the web 2.0 environment and social networks. Group Decision and Negotiation, 17, 387-402. doi:10.1007/s10726008-9113-2.

Lassila, O., \& Hendler, J. (2007). Embracing "web 3.0". IEEE Internet Computing, 11, 90-93.

Muysken, P. (2000). Bilingual speech: A typology of code-mixing: Cambridge University Press.

Nagle, T., \& Pope, A. (2013). Understanding social media business value, a prerequisite for social media selection. Journal of Decision Systems, 22, 283-297. doi:10.1080/ 12460125.2014 .846543$.

Necula, S.- C. (2011). Discussions on semantic-based in decision support systems. In 5th European conference on European computing conference (ECC'11) (pp. 193-197). Paris, France: World Scientific and Engineering Academy and Society (WSEAS).

Necula, S.-C. (2012). Implementing the main functionalities required by semantic search in decision-support systems. International Journal of Computers, Communications \& Control (IJCCC), 7, 907-915 


\section{AQ10}

Nonaka, I., \& Takeuchi, H. (1995). The knowledge creating company: How Japanese companies create the dynamics of innovation: Oxford University Press.

O'Reilly, T. (2005, September 05). What is web 2.0? - design patterns and business models for the next generation of software. Retrieved from http://oreilly.com/web2/archive/what-is-web20.html

O'Reilly, T. (2006, December 06). Web 2.0 compact definition: Trying again. Retrieved from http://radar.oreilly.com/2006/12/web-20-compact-definition-tryi.html

Padula, M., Reggiori, A., \& Capetti, G. (2009). Managing collective knowledge in the web 3.0. In I. C. Society (Ed.), INTERNET 2009, First International Conference on Evolving Internet. Cannes/La Bocca, French Riviera, France.

Pattal, M. M. I., Yuan, L., \& Jianqiu, Z. (2009). Web 3.0: A real personal web! more opportunities \& more threats. In Third International Conference on Next Generation Mobile Applications, Services and Technologies. Cardiff, Wales, UK: IEEE Computer Society Press.

Power, D. J., \& Phillips-Wren, G. (2011). Impact of social media and web 2.0 on decision-making. Journal of Decision Systems, 20, 249-261. doi:10.3166/jds.20.249-261.

Power, D. J., Sharda, R., \& Kulkarni, U. R. (2007). Understanding decision support systems for global enterprises. Decision Support for Global Enterprises, (Vol. 2, 3-14): Springer US.

Richter, D., Riemer, K., \& Brocke, J. V. (2011). Internet social networking: Research state of the art and implications for enterprise 2.0. Business \& Information Systems Engineering, 2, 89-101. doi:10.1007/s12599-011-0151-y.

Robu, V., Halpin, H., \& Shepherd, H. (2009). Emergence of consensus and shared vocabularies in collaborative tagging systems. ACM Transactions on the Web (TWEB), 3, Article 14. doi: $10.1145 / 1594173.1594176$

Rodriguez, M. A., Steinbock, D. J., Watkins, J. H., Gershenson, C., Bollen, J., Grey, V., \& deGraf, B. (2007). Smartocracy: Social networks for collective decision making. In 40th Hawaii International Conference on System Sciences (CD-ROM). Computer Society Press.

Russell, S. J., \& Norvig, P. (2010). Artificial Intelligence: A Modern Approach (3rd ed.). Upper Saddle River, New Jersey: Pearson Education Inc.

Schneider, J., Groza, T., \& Passant, A. (2013). A review of argumentation for the social semantic web. Semantic Web, 4, 159-218. doi:10.3233/SW-2012-0073.

Shum, S. B., Cannavacciuolo, L., De Liddo, A., Iandoli, L., \& Quinto, I. (2011). Using social network analysis to support collective decision-making process. International Journal of Decision Support System Technology, 3, 15-31.

Simon, H. A. (1977). The new science of management decision. Englewoods Cliffs, NJ: Prentice Hall.

Surowiecky, J. (2004). The wisdom of crowds: Why the many are smarter than the few and how collective wisdom shapes business. In Economies, Societies and Nations (Reprint ed., pp. 3-22). New York, NY: Anchor.

Turban, E., Aronson, J. E., \& Liang, T. P. (2005). Decision support systems and intelligent systems (7th ed.). New Jersey: Pearson Education Inc.

Turban, E., Liang, T.-P., \& Wu, S. P. J. (2011). A framework for adopting collaboration 2.0 tools for virtual group decision making. Group Decision and Negotiation, 20, 137-154. doi:10.1007/s10726-010-9215-5.

Venkatesh, V., \& Davis, F. D. (2000). A theoretical extension of the technology acceptance model: Four longitudinal field studies. Management Science, 46, 186-204. 